\title{
The limb darkening of $\alpha$ Centauri B
}

\section{Matching 3D hydrodynamical models with interferometric measurements}

\author{
L. Bigot ${ }^{1}$, P. Kervella ${ }^{2}$, F. Thévenin ${ }^{1}$, and D. Ségransan ${ }^{3}$ \\ 1 Département Cassiopée, UMR 6202, Observatoire de la Côte d'Azur, BP 4229, 06304 Nice Cedex 4, France \\ e-mail: lbigot@obs-nice.fr \\ 2 LESIA, UMR 8109, Observatoire de Paris-Meudon, 5 place Jules Janssen, 92195 Meudon Cedex, France \\ 3 Observatoire de Genève, 1290 Sauverny, Switzerland
}

Received 4 April 2005 / Accepted 12 September 2005

ABSTRACT

For the nearby dwarf star $\alpha$ Cen B (K1 V), we present limb-darkening predictions from a 3D hydrodynamical radiative transfer model of its atmosphere. We first compared the results of this model to a standard Kurucz's atmosphere. Then we used both predictions to fit the new interferometric visibility measurements of $\alpha$ Cen B obtained with the VINCI instrument of the VLT Interferometer. Part of these new visibility measurements were obtained in the second lobe of the visibility function, which is sensitive to stellar limb-darkening. The best agreement is found for the 3D atmosphere limb-darkening model and a limb-darkened angular diameter of $\theta_{3 \mathrm{D}}=6.000 \pm 0.021$ mas, corresponding to a linear radius of $0.863 \pm 0.003 R_{\odot}$ (assuming $\pi=747.1 \pm 1.2$ mas). Our new linear radius agrees well with the asteroseismic value predicted by Thévenin et al. (2002, A\&A, 392, L9). In view of future observations of this star with the VLTI/AMBER instrument, we also present limb-darkening predictions in the $J, H$, and $K$ bands.

Key words. instrumentation: interferometers - stars: atmospheres

\section{Introduction}

Limb-darkening (hereafter LD) is a well-known effect in stellar physics. Its manifestation is a non-uniform brightness of the disk, whose edges appear fainter than the center. This effect occurs because of the decrease in the source function outwards in the atmosphere. The disk center then shows deeper and warmer layers, whereas the edges show higher and cooler material. This means that analysis of the intensity $I_{\lambda}(\mu)$ at different latitudinal angles $\mu=\cos \theta$ provides information on the temperature variation with depth in the external layers of the star. This is therefore an excellent constraint for testing atmospheric models, to validate or invalidate assumptions used to derive these models (like NLTE/LTE), and to provide suggestions for improving the input physics (equation-of-state and/or opacities in particular). The center-to-limb variation of the Sun has been known for many years and been measured for numerous $\mu$ and $\lambda$ (e.g. Pierce \& Slaughter 1977; Neckel \& Labs 1994; Hestroffer \& Magnan 1998) leading to a plethora of theoretical works that have improved our knowledge of the external layers of the Sun.

Traditionally, analysis of solar and stellar LD is made by adopting an approximated law for $I_{\lambda}(\mu)$, generally a polynomial expansion in $\mu$ that are either linear or non-linear (see e.g. Claret 2000, for recent developments) and with coefficients determined from 1D atmospheric models, like ATLAS
(Kurucz 1992) or Phoenix (Hauschildt 1999). However, in spite of the detailed physics included in these codes, their 1D nature is a limitation for deriving realistic emergent intensities. Indeed, these codes contain free parameters, like the wellknown mixing length parameter, which are injected artificially in order to reproduce the properties of the turbulent convection at the stellar surface. As a consequence, the comparison between these 1D models and observations depends on the input parameters, which thereby creates an important source of uncertainties. Moreover, convection is by nature a 3D process. Its manifestation is the presence of bright granules and dark intergranular lanes. Reducing it to a $1 \mathrm{D}$ process, i.e. ignoring horizontal flows and temperature inhomogeneities, changes the pressure scale height, the location of the surface, and therefore also the emergent intensity (see e.g. Allende-Prieto et al. 2004; and Asplund et al. 2000a, for a comparison of multidimensional simulations).

The precise measurements of the center-to-limb variation achieved nowadays require realistic stellar atmospheric models that take all the complexity of the stellar surface into account, and motivates the use of the new generation of $3 \mathrm{D}$ radiative hydrodynamical (hereafter RHD) simulations.

In this paper we propose a study of $\alpha$ Cen B (HD128621), a nearby K1V dwarf star. It is part of a visual triple star 
system whose brightest component, $\alpha$ Cen A (HD128620), is a G2V dwarf. Both theoretical and observational considerations motivated the selection of this star in the present work. From the interferometric point of view, the proximity $(1.3 \mathrm{pc})$ of the star is a rare opportunity for interferometric measurements, since most of the nearby dwarfs have angular diameters that are too small to be measured. Our interest in this star grew recently since our new measurements provided data points in the second lobe of the visibility function, which is sensitive to the LD of the star. From a theoretical point of view, this star is important for various reasons. In particular, recent detection of solar-like oscillations in $\alpha$ Cen A and B (Bouchy \& Carrier 2001, 2002; Carrier \& Bourban 2003) have led several authors (e.g. Morel et al. 2000; Thévenin et al. 2002; Thoul et al. 2004; Eggenberger et al. 2004) to build evolution models of these two stars that are strongly constrained by the measured frequency spacings. The result is a better, but still debated, determination of the fundamental parameters of the system.

In Sect. 2, we report the new interferometric measurements of $\alpha$ Cen B obtained since 2003 using the VINCI instrument. Section 3 describes our 3D simulations to derive self-consistent stellar limb-darkening of $\alpha$ Cen B. They are subsequently used to compute visibility curves in the near-infrared (Sect. 4) in order to interpret our measurements in terms of stellar angular diameter and to discuss the agreement between the 3D limbdarkening model and our second lobe visibility measurements. We also use our simulations to predict future observations $(J$, $H$, and $K$ bands) that will be made with the next generation of instruments of the VLTI, such as the new AMBER instrument (Petrov et al. 2000; Robbe-Dubois et al. 2004).

\section{New interferometric observations}

A total of 37 new interferometric measurements of $\alpha$ Cen B were obtained in 2003 on two baselines, D1-B3 (24 m in ground length) and B3-M0 $(140 \mathrm{~m})$, using the VINCI instrument (Kervella et al. 2000; Kervella et al. 2003a). The points obtained on the longer baseline are located in the second lobe of the visibility function of $\alpha \mathrm{Cen} \mathrm{B}$, whose shape depends on the limb-darkening. We obtained 1000 interferograms on the B3-M0 baseline in two series. Out of these, 534 were processed by the VINCI pipeline. The B3-M0 baseline observations are made difficult by the very low $V^{2}$ of the interferometric fringes, less than $2 \%$. However, Fig. 1 shows an example of the power spectral density of these very low visibility fringes where no bias is present. On the D1-B3 baseline, we recorded 17500 interferograms in 35 series (15 141 processed). These new measurements were added to the $V^{2}$ values obtained on the E0-G1 baseline and have already been published in Kervella et al. (2003b, hereafter Paper I). The resulting squared visibilities are listed in Tables 2 and 3.

We used several stars from the Cohen et al. (1999) catalog as calibrators to estimate the point source response of the interferometer. They were observed immediately before or after $\alpha$ Cen B. On the D1-B3 baseline, we used HD 119193 $\left(\theta_{\mathrm{UD}}=2.03 \pm 0.022 \mathrm{mas}\right), 58 \mathrm{Hya}\left(\theta_{\mathrm{UD}}=3.13 \pm 0.030 \mathrm{mas}\right)$ and HD $112213\left(\theta_{\mathrm{UD}}=3.14 \pm 0.025 \mathrm{mas}\right)$. Approximately one third of the measurements were obtained with each of these

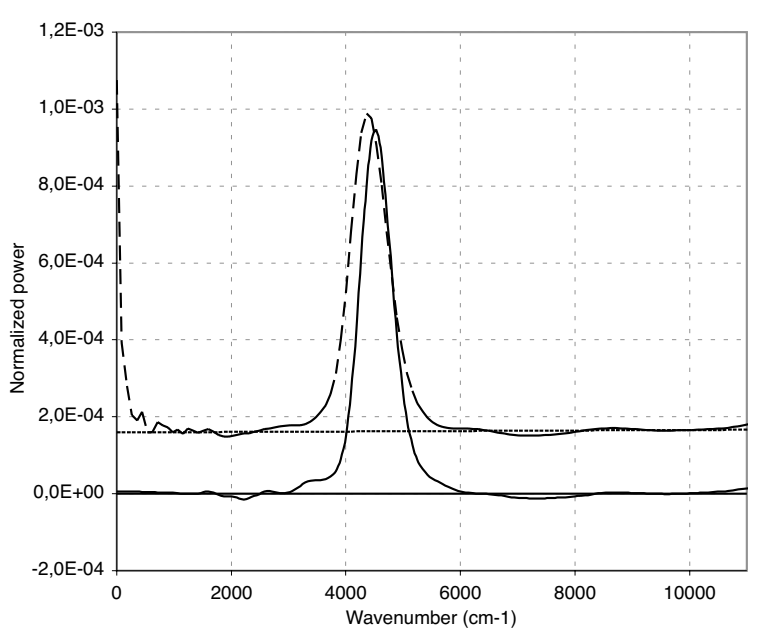

Fig. 1. The average wavelet power spectral density (WPSD) of 299 interferograms of $\alpha$ Cen B obtained on JD 2452770.6605 (11 May 2003). In spite of the very low visibility $\left(V^{2}=1.38 \%\right)$, subtraction of the background noise (dotted line) from the processed fringe's power peak (dashed line) leaves no residual bias on the final WPSD (solid line). The power integration is done between wave numbers 1970 and $7950 \mathrm{~cm}^{-1}$.

calibrators. On the B3-M0 baseline, we relied on HR 4831 $\left(\theta_{\mathrm{UD}}=1.66 \pm 0.018 \mathrm{mas}\right)$, whose small size results in a low systematic uncertainty on the calibrated $V^{2}$ values. The angular diameter estimates from Cohen et al. (1999) have been verified by Bordé et al. (2002) as reliable within their stated error bars. The squared visibilities were derived using the processing methods described in Kervella et al. (2004). As an example, the calibration sequence used for the longest baseline B3-M0 is presented in Table 1 .

\section{Simulation of a 3D atmosphere}

In order to model the intensity profile of $\alpha \mathrm{Cen} \mathrm{B}$, we performed realistic, time-dependent, 3D radiative hydrodynamical simulations of its surface. The emerging intensity of the atmospheric model in different directions was used to build theoretical monochromatic limb-darkening profiles for various wavelengths covering the spectral domains of interest for the VINCI and AMBER instruments (in the 1.0-2.4 $\mu \mathrm{m}$ range).

\subsection{The stellar atmosphere modelling}

The numerical code used for this work belongs to a new generation of 3D atmospheric codes developed for the study of solar (e.g. Stein \& Nordlund 1989, 1998) and stellar (e.g. Nordlund \& Dravins 1990; Asplund et al. 2000; Allende-Prieto et al. 2002; Ludwig et al. 2002) granulation and line formations (e.g. Asplund et al. 2000a,b,c, 2004, 2005). The code solves the non-linear, compressible equations of mass, momentum, and energy conservation on a Eulerian mesh. The 3D radiative transfer was solved at each time step along different inclined rays for which we assumed local thermodynamical equilibrium (LTE). We considered 10 latitudinal $\mu$ points and 4 longitudinal $\varphi$ points, and checked that a finer grid in $(\mu, \varphi)$ does not change the properties of the model. Realistic equation-of-state 
Table 1. Calibration sequence of $\alpha$ Cen B on the B3-M0 baseline ( $140 \mathrm{~m}$ ground length). The expected visibilities $V_{\text {theo }}^{2}$ given in this table include the bandwidth smearing effect. The interferometric efficiency given in italics corresponds to the value assumed for the calibration of these particular $\alpha$ Cen B observations (see Tables 2 and 3). The HR 4831 data marked with $\left(^{*}\right.$ ) were taken $2 \mathrm{~h}$ before $\alpha$ Cen B, and are listed to show the stability of the IE, but were not used for the IE estimation.

\begin{tabular}{cccccccl}
\hline \hline JD & Scans & $B(\mathrm{~m})$ & Azim. & $\mu^{2} \pm$ stat. $(\%)$ & $V_{\text {theo }}^{2} \pm$ syst. $(\%)$ & $I E \pm$ stat. \pm syst. (\%) & Target \\
\hline 2452770.5474 & 365 & 139.309 & 49.24 & $29.37 \pm 0.39$ & $49.93 \pm 0.80$ & $58.83 \pm 0.79 \pm 0.94^{*}$ & HR 4831* $^{*}$ \\
2452770.5523 & 316 & 139.131 & 50.51 & $29.38 \pm 0.51$ & $50.06 \pm 0.80$ & $58.70 \pm 1.02 \pm 0.93^{*}$ & HR 4831* $^{*}$ \\
2452770.5572 & 296 & 138.913 & 51.77 & $30.05 \pm 0.67$ & $50.19 \pm 0.80$ & $59.88 \pm 1.34 \pm 0.95^{*}$ & HR 4831* $^{*}$ \\
\hline 2452770.6368 & 405 & 128.934 & 71.28 & $32.05 \pm 0.35$ & $55.48 \pm 0.75$ & $57.78 \pm 0.62 \pm 0.77$ & HR 4831 \\
2452770.6419 & 408 & 127.841 & 72.49 & $31.60 \pm 0.36$ & $56.01 \pm 0.74$ & $56.42 \pm 0.64 \pm 0.74$ & HR 4831 \\
2452770.6469 & 392 & 126.698 & 73.69 & $32.72 \pm 0.36$ & $56.54 \pm 0.73$ & $57.87 \pm 0.64 \pm 0.74$ & HR 4831 \\
\hline 2452770.6605 & 299 & 133.838 & 59.85 & $0.791 \pm 0.039$ & & $57.36 \pm 0.82 \pm 0.74$ & $\alpha$ Cen B \\
2452770.6656 & 235 & 133.277 & 61.33 & $0.777 \pm 0.082$ & & $57.36 \pm 0.82 \pm 0.74$ & $\alpha$ Cen B \\
\hline
\end{tabular}

Table 2. $\alpha$ Cen B squared visibilities.

\begin{tabular}{lccc}
\hline \hline JD & $B(\mathrm{~m})$ & Azim. & $V^{2} \pm$ stat. \pm syst. $(\%)$ \\
\hline D1-B3 & & & \\
2452720.9141 & 20.891 & 108.36 & $81.24 \pm 3.03 \pm 0.09$ \\
2452720.9081 & 21.095 & 106.30 & $83.40 \pm 2.80 \pm 0.09$ \\
2452725.8927 & 21.152 & 105.72 & $80.48 \pm 1.83 \pm 0.04$ \\
2452720.9029 & 21.270 & 104.52 & $83.89 \pm 2.66 \pm 0.09$ \\
2452725.8878 & 21.315 & 104.05 & $79.99 \pm 1.81 \pm 0.04$ \\
2452725.8828 & 21.479 & 102.36 & $80.95 \pm 1.84 \pm 0.04$ \\
2452720.8627 & 22.462 & 91.40 & $83.75 \pm 3.84 \pm 0.10$ \\
2452725.8408 & 22.669 & 88.80 & $79.26 \pm 2.54 \pm 0.06$ \\
2452725.8358 & 22.786 & 87.24 & $78.79 \pm 2.52 \pm 0.06$ \\
2452720.8489 & 22.799 & 87.08 & $82.95 \pm 3.88 \pm 0.10$ \\
2452725.8306 & 22.903 & 85.63 & $79.32 \pm 2.54 \pm 0.06$ \\
2452720.8434 & 22.921 & 85.36 & $82.06 \pm 3.76 \pm 0.10$ \\
2452726.8032 & 23.375 & 77.99 & $77.19 \pm 0.64 \pm 0.07$ \\
2452703.8642 & 23.405 & 77.43 & $80.79 \pm 2.25 \pm 0.05$ \\
2452726.7983 & 23.452 & 76.49 & $78.82 \pm 0.60 \pm 0.07$ \\
2452703.8599 & 23.470 & 76.13 & $80.05 \pm 2.23 \pm 0.05$ \\
2452726.7933 & 23.525 & 74.96 & $77.69 \pm 0.59 \pm 0.07$ \\
2452703.8555 & 23.534 & 74.77 & $81.05 \pm 2.23 \pm 0.05$ \\
2452723.7937 & 23.627 & 72.58 & $77.60 \pm 0.93 \pm 0.10$ \\
2452723.7885 & 23.688 & 71.00 & $77.11 \pm 0.86 \pm 0.10$ \\
2452723.7835 & 23.741 & 69.46 & $78.76 \pm 0.81 \pm 0.10$ \\
2452723.7521 & 23.953 & 59.81 & $78.42 \pm 0.77 \pm 0.10$ \\
2452703.8019 & 23.970 & 58.32 & $79.61 \pm 2.50 \pm 0.05$ \\
2452723.7469 & 23.970 & 58.20 & $78.20 \pm 0.83 \pm 0.10$ \\
2452704.7984 & 23.971 & 58.09 & $81.26 \pm 0.98 \pm 0.05$ \\
2452703.7979 & 23.980 & 57.06 & $80.38 \pm 2.46 \pm 0.05$ \\
2452704.7940 & 23.982 & 56.70 & $81.38 \pm 0.97 \pm 0.05$ \\
2452723.7419 & 23.982 & 56.63 & $77.93 \pm 0.55 \pm 0.10$ \\
2452709.7555 & 23.985 & 48.87 & $82.93 \pm 3.59 \pm 0.10$ \\
2452704.7896 & 23.989 & 55.34 & $80.51 \pm 1.01 \pm 0.05$ \\
2452716.7402 & 23.990 & 50.10 & $77.55 \pm 3.22 \pm 0.08$ \\
2452709.7596 & 23.991 & 50.19 & $81.43 \pm 3.55 \pm 0.10$ \\
2452726.7251 & 23.994 & 53.95 & $76.95 \pm 0.90 \pm 0.08$ \\
2452716.7448 & 23.994 & 51.55 & $76.31 \pm 3.22 \pm 0.08$ \\
2452709.7640 & 23.994 & 51.57 & $77.31 \pm 3.73 \pm 0.09$ \\
\hline & & &
\end{tabular}

(including ionization, dissociation, and recombination) and opacities (Uppsala opacity package, Gustafsson et al. 1975) were used. The line-blanketing was taken into account through
Table 3. $\alpha$ Cen B squared visibilities (continued).

\begin{tabular}{lccc}
\hline \hline JD & $B(\mathrm{~m})$ & Azim. & $V^{2} \pm$ stat. \pm syst. $(\%)$ \\
\hline E0-G1* & & & \\
2452462.5836 & 60.441 & 157.57 & $17.02 \pm 0.36 \pm 0.26$ \\
2452462.5870 & 60.544 & 158.40 & $17.01 \pm 0.23 \pm 0.26$ \\
2452462.5905 & 60.645 & 159.26 & $16.80 \pm 0.77 \pm 0.26$ \\
2452462.5949 & 60.767 & 160.35 & $16.05 \pm 0.68 \pm 0.24$ \\
2452465.6268 & 61.541 & 170.27 & $16.76 \pm 1.05 \pm 0.26$ \\
2452470.6203 & 61.621 & 172.05 & $14.94 \pm 0.44 \pm 0.23$ \\
2452470.6234 & 61.650 & 172.82 & $15.59 \pm 0.42 \pm 0.24$ \\
2452470.6278 & 61.687 & 173.92 & $16.70 \pm 0.44 \pm 0.25$ \\
\hline B3-M0 & & & \\
2452770.6605 & 133.838 & 59.85 & $1.379 \pm 0.07 \pm 0.02$ \\
2452770.6656 & 133.277 & 61.33 & $1.356 \pm 0.14 \pm 0.02$ \\
\hline
\end{tabular}

* E0-G1 measurements reported by Kervella et al. (2003b).

the opacity binning technique (Nordlund 1982). In the present simulation we considered a Cartesian grid of $(x, y, z)=125 \times$ $125 \times 82$ points. The geometrical sizes are $6 \times 6 \mathrm{Mm}$ for the horizontal directions and $5 \mathrm{Mm}$ for the vertical one. The dimensions of this domain are large enough to include a sufficiently large number of granules $(n \geq 20)$ simultaneously, which prevents statistical bias. A periodic boundary condition was applied for the horizontal directions, and transmitting vertical boundaries were used at the top and bottom of the domain. The base of the domain was adjusted to have a nearly adiabatic, isentropic, and featureless convective transport. The upper boundary was placed sufficiently high in the atmosphere so that it does not influence the property of the model.

A detailed description of the current version of the code used in this paper may be found in Stein \& Nordlund (1998). Unlike 1D hydrostatic models that reduce all hydrodynamics to a single adjustable parameter, the present simulations were done ab initio by solving the complete set of RHD equations in a self-consistent way. All the dynamics and turbulence of the model came naturally from the equations of physics. Nothing was adjusted, such as the convective flux in the MLT formalism. The diagnostic made by such RHD simulations is, therefore, much more realistic than the 1D models. We emphasize that the realism of these 3D simulations has been intensively 
Table 4. limb-darkening $I(\lambda, \mu)$ for various wavelengths over the $J H K$ range.

\begin{tabular}{cccccccccccc}
\hline \hline$\lambda(\mu \mathrm{m}) / \mu$ & 0.0 & 0.1 & 0.2 & 0.3 & 0.4 & 0.5 & 0.6 & 0.7 & 0.8 & 0.9 & 1.0 \\
\hline 1.050 & 0.4434 & 0.5745 & 0.6453 & 0.7069 & 0.7605 & 0.8087 & 0.8527 & 0.8932 & 0.9311 & 0.9667 & 1.0000 \\
1.270 & 0.4646 & 0.6017 & 0.6738 & 0.7347 & 0.7860 & 0.8310 & 0.8711 & 0.9074 & 0.9406 & 0.9715 & 1.0000 \\
1.650 & 0.4838 & 0.6752 & 0.7487 & 0.8039 & 0.8462 & 0.8812 & 0.9110 & 0.9369 & 0.9601 & 0.9811 & 1.0000 \\
2.000 & 0.5442 & 0.7063 & 0.7707 & 0.8202 & 0.8585 & 0.8905 & 0.9178 & 0.9417 & 0.9630 & 0.9825 & 1.0000 \\
2.200 & 0.5729 & 0.7220 & 0.7817 & 0.8283 & 0.8646 & 0.8950 & 0.9211 & 0.9439 & 0.9645 & 0.9831 & 1.0000 \\
2.400 & 0.5968 & 0.7353 & 0.7912 & 0.8352 & 0.8698 & 0.8988 & 0.9239 & 0.9458 & 0.9656 & 0.9836 & 1.0000 \\
\hline
\end{tabular}

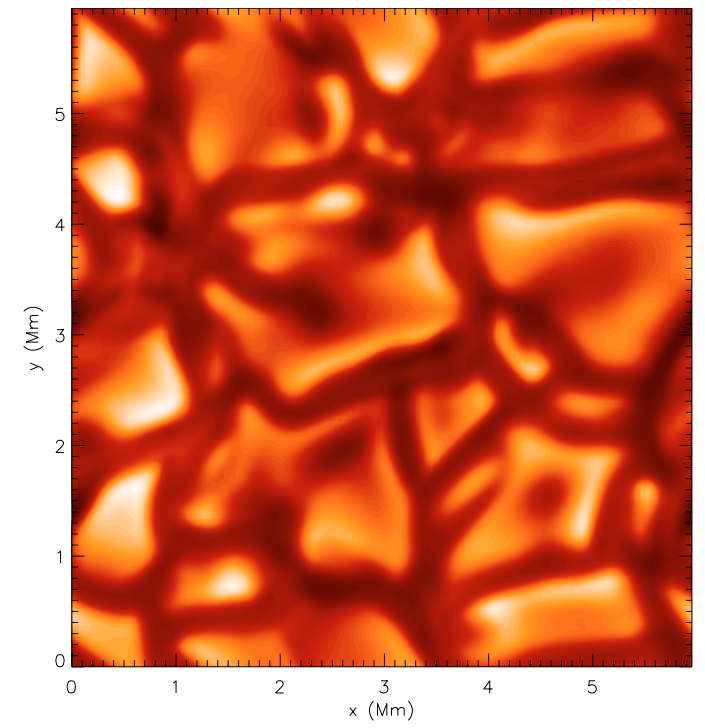

Fig. 2. Snapshot of the disk-center $(\mu=1)$ intensity emerging at the stellar surface at a representative time.

checked for solar line formations (e.g. Asplund et al. 2000b,c, 2004), helioseismology (e.g. Rosenthal et al. 1999), and also for stellar line formations (e.g. Allende-Prieto et al. 2002).

The adopted atmospheric parameters are those of Morel et al. (2000), i.e. $T_{\text {eff }}=5260 \mathrm{~K}, \log g=4.51$ and $[\mathrm{Fe} / \mathrm{H}]=+0.2$. The simulation was run for a few hours of stellar time that covered several convective turn-over times. The result is a $3 \mathrm{D}$, time-dependent box representing the stellar surface. A snapshot of the disk-center surface intensity is represented in Fig. 2. The structure of our model is similar to the one obtained by Nordlund \& Dravins (1990) but is even more realistic, since the present version of the code solves compressible equations of hydrodynamics and uses more grid-points, which allows a better treatment of the turbulence.

\section{2. $3 D$ limb-darkening}

The monochromatic surface intensity was computed for various latitudinal $\mu$ and longitudinal $\varphi$ directions at the stellar surface. The limb-darkening $I_{\lambda}(\mu)$ was obtained by horizontal $(x, y)$, longitudinal and time averages of the surface intensity. For the time average, we considered a sequence of 2 hours of stellar time. The results are plotted in Fig. 3 for the two extreme wavelengths of our spectral domain, 1.0 and $2.4 \mu \mathrm{m}$. For comparison, we overplot limb-darkening obtained from a 1D ATLAS9 model for the same wavelengths and for the same stellar fundamental parameters. It appears that 3D RHD produces a less significant center-to-limb variation than a 1D static model. The departure from a $1 \mathrm{D}$ model increases with decreasing wavelengths. Such behavior was also found by Allende Prieto et al. (2002) for Procyon. However, in the case of $\alpha$ Cen $\mathrm{B}$, the departure from 1D to 3D limbdarkening is smaller, as a consequence of a less efficient convection in $\mathrm{K}$ dwarfs as compared to $\mathrm{F}$ stars.

The reason the emergent intensity differs between 1D and $3 \mathrm{D}$ models is that the properties of the superadiabatic and surface convective layers cannot be described well by the mixing length formalism, whatever parameter we choose. The temperature inhomogeneities (granulation) together with the strong sensitivity of the opacity $\left(\mathrm{H}^{-}\right)$to the temperature make the warm ascending flows more opaque than they would be for a homogeneous $1 \mathrm{D}$ model. This purely $3 \mathrm{D}$ effect, added to the contribution of the turbulent pressure, pushes the location of the surface to lower densities. The temperature gradient in these regions is steeper than in the $1 \mathrm{D}$ case (see Nordlund \& Dravins 1990). Since the continuum is formed in these layers, the emergent intensity is different.

The correction due to 3D simulations (a few percents) is small but not negligible with respect to the precision obtained by the new generation of interferometric instruments like VINCI or AMBER. This improvement is essential for deriving an accurate angular diameter of the star. We report our limb-darkening predictions in Table 4 for a series of continuum wavelengths between 1.0 and $2.4 \mu \mathrm{m}$, corresponding to the $J H K$ range accessible to the AMBER instrument.

\section{Visibility model and angular diameter of $\alpha$ Cen B}

In this section, we describe the application of our 3D limbdarkening models to the interpretation of the VINCI measurements of $\alpha$ Cen B.

\subsection{Limb-darkened disk visibility model}

In the simple case of a centro-symmetric star such as $\alpha$ Cen B, the visibility function measured using a broadband interferometric instrument such as VINCI is defined by three wavelength-dependent functions:

1. The spectral energy distribution $S(\lambda)$ of the star, expressed in terms of photons (VINCI uses a photon counting detector). 

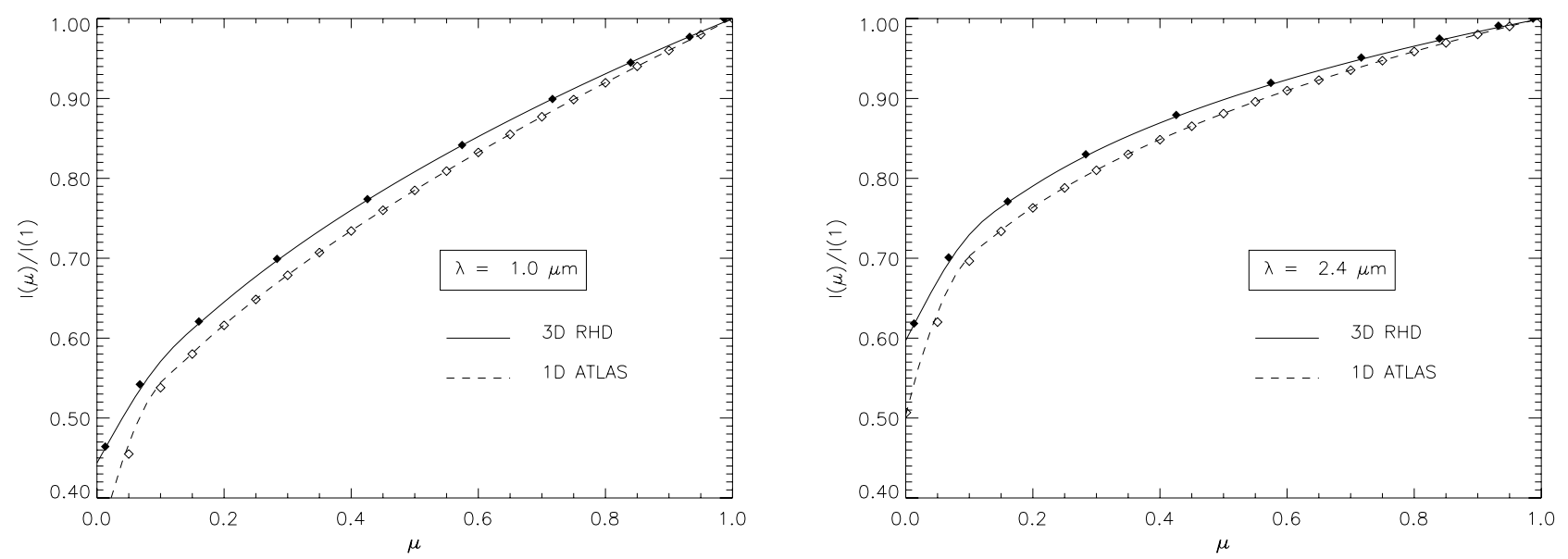

Fig. 3. Normalized monochromatic center-to-limb variation $I_{\lambda}(\mu) / I_{\lambda}(1)$ of the surface intensity obtained by 3D RHD simulations of $\alpha$ Cen B as a function of $\mu$ for two different wavelengths: $1 \mu \mathrm{m}$ (left panel) and $2.4 \mu \mathrm{m}$ (right panel), which correspond to the extreme limits of the spectral domain we have considered in this paper. The solid lines represent the 3D RHD limb-darkening, whereas the dashed lines represent limb darkening derived from 1D ATLAS atmospheric models. In both case, the symbols $\diamond$ represent the values extracted from both 1D (white) and 3D (black) simulations.

2. The wavelength-dependent intensity profile of the star: $I(\lambda, \mu) / I(\lambda, 1)$.

3. The spectral transmission $T(\lambda)$ of the instrument, including the atmosphere, all optical elements and the detector quantum efficiency.

Out of these three functions, $T(\lambda)$ is known from the conception of the instrument, as well as from calibrations obtained on the sky (see Kervella et al. 2003b, for details). The spectral energy distribution of the star $S(\lambda)$ can be measured directly using a spectrograph or taken from atmospheric numerical models.

From the 3D RHD simulations presented in Sect. 3, we obtained intensity profiles for ten distinct wavelengths over the $K$ band (chosen in the continuum). For each of these profiles, ten values of $\mu$ were computed. The resulting $10 \times 10$ element 2D table $I(\lambda, \mu) / I(\lambda, 1)$ was then interpolated to a larger $60 \times 50$ element table, with a $10 \mathrm{~nm}$ step in wavelength (over the $1.90-2.50 \mu \mathrm{m}$ range) and a 0.02 step in $\mu$. This interpolation preserves the smooth shape of the intensity profile function well. This procedure was also used to build the $I(\lambda, \mu) / I(\lambda, 1)$ table based on the 1D Kurucz model. The original sample $(10 \times 20)$ was interpolated to the same final grid as the $3 \mathrm{D}$ model.

We can derive the monochromatic visibility law $V_{\lambda}(B, \theta)$ from the monochromatic intensity profile $I(\lambda, \mu)$ using the Hankel integral:

$V_{\lambda}(B, \theta)=\frac{1}{A} \int_{0}^{1} I(\lambda, \mu) J_{0}\left(\frac{\pi B \theta_{\mathrm{LD}}}{\lambda} \sqrt{1-\mu^{2}}\right) \mu \mathrm{d} \mu$,

where $B$ is the baseline (in meters), $\theta$ the limb darkened angular diameter (in radians), $J_{0}$ the zeroth order of the Bessel function, $\lambda$ the wavelength (in meters), $\mu=\cos \theta$ the cosine of the azimuth of a surface element of the star, and $A$ the normalization factor:

$A=\int_{0}^{1} I(\lambda, \mu) \mu \mathrm{d} \mu$.
To obtain the visibility function observed by VINCI in broadband, we have to integrate this function taking the transmission of the instrument and the spectral energy distribution of the star into account:

$V_{K}(B, \theta)=\frac{\int_{K}\left[V_{\lambda}(B, \theta) T(\lambda) S(\lambda)\right]^{2} \lambda^{2} \mathrm{~d} \lambda}{\int_{K}[T(\lambda) S(\lambda)]^{2} \lambda^{2} \mathrm{~d} \lambda}$.

Note the $\lambda^{2}$ term that is necessary, as the actual integration of the squared visibility by VINCI over the $K$ band is done in the Fourier conjugate space of the optical path difference (expressed in meters), and is therefore done in wavenumber $\sigma=1 / \lambda$. This corrective term ensures that the integration of the star's spectrum is done precisely in the same way as in the instrument.

This formulation is very general, as it does not make any particular assumption about the spectrum of the star or about the wavelength dependence of its intensity profile $I(\lambda, \mu)$.

\subsection{Fit of the interferometric data and angular diameter of $\alpha$ Cen $B$}

Considering the model discussed in Sect. 4.1, we now derive the limb-darkened angular diameter $\theta_{\mathrm{LD}}$ of $\alpha$ Cen B. It is obtained by a standard $\chi^{2}$ analysis of the data. We define the reduced $\chi^{2}$ of our fit by

$\chi_{\text {red }}^{2}=\frac{1}{N-n} \sum_{i=1}^{N}\left(\frac{V_{i}^{2}-V_{\text {model }}^{2}}{\sigma_{i}}\right)^{2}$,

where $n$ is the number of variables ( $n=1$ for our fit), $N$ the total number of measurements, $i$ the index of a particular measurement, and $\sigma_{i}$ the standard deviation of the measurement with index $i$.

The $\chi^{2}$ minimization was computed for three center-to-limb models: uniform disk (UD), 1D ATLAS, and 3D RHD. In each case, the broadband square visibility curve $V_{K}^{2}(B, \theta)$ is shown in Figs. 4 and 5. In addition to the purely statistical error, we must 


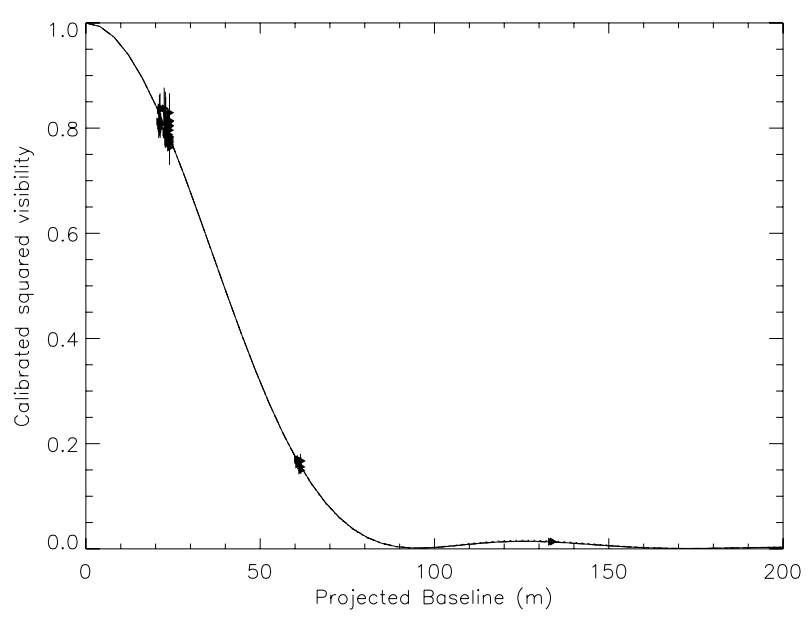

Fig. 4. Overview of $\alpha$ Cen B squared visibilities. The continuous line represents the broadband, limb darkened disk visibility model derived from the 3D RHD with $\theta_{3 \mathrm{D}}=6.000$ mas.

also take two systematic error sources into account: the calibration uncertainty and the wavelength uncertainty. The calibration uncertainty comes from the errors on the a priori angular sizes of the five calibrators that were used for the VINCI observations. It amounts to 0.012 mas on the final angular diameter. The wavelength uncertainty comes from the imperfect knowledge of the transmission of VINCI, in particular of its fluoride glass optical fibers. This transmission was calibrated on the sky (Paper I), and the uncertainty on this measurement is estimated to be $0.15 \%$. As it impacts linearly on the angular diameter value, it corresponds to 0.009 mas. These two systematic factors add up quadratically to the 0.013 mas statistical uncertainty and result in a total error of 0.021 mas on the angular diameters of $\alpha \mathrm{Cen} \mathrm{B}$. The best fit angular diameter that we derive using our 3D limb-darkening model is $\theta_{3 \mathrm{D}}=$ $6.000 \pm 0.021$ mas. The $1 \mathrm{D}$ model produces a slightly larger diameter, $\theta_{1 \mathrm{D}}=6.017 \pm 0.021 \mathrm{mas}$, and the UD disk produces naturally a much smaller diameter, $\theta_{\mathrm{UD}}=5.881 \pm 0.021$ mas.

There is no significant difference between the three models in the first lobe of visibility. However, different amplitudes of the second lobe were observed. While the UD model produces higher visibilities, the 1D limb-darkened model leads to visibilities that are slightly too low compared to our observations. Overall, the 3D model leads to a slightly better agreement with observations.

As expected, the difference $3 \mathrm{D} / 1 \mathrm{D}$ is rather small, since we are working in the near-infrared ( $K$-band) and with a dwarf star. It is nonetheless comparable to $\sigma_{\text {stat }}$ and therefore significant.

\subsection{Linear diameter}

Assuming the parallax value of Söderhjelm (1999), $\pi=747.1 \pm$ 1.2 mas $^{1}$, we found a linear radius of $0.863 \pm 0.003 R_{\odot}$ which agrees with results presented in Paper 1. We estimated that the

\footnotetext{
${ }^{1}$ One should note that there is a rather broad distribution of parallax values for $\alpha$ Cen in the literature, as discussed in Paper I. While the value from Söderhjelm (1999) is the result of a careful reprocessing of the Hipparcos data, the possibility of a bias beyond the stated $1 \sigma$
}

adopted uncertainty in $T_{\text {eff }}(=50 \mathrm{~K})$ leads to an error of about $0.0003 R_{\odot}$, i.e. ten times smaller than the derived uncertainties. From the $1 \mathrm{D}$ analysis, we derived a radius of $0.865 \pm 0.003 R_{\odot}$, larger than the radius found by the RHD approach by about $1 \sigma_{\text {stat }}$. In addition to the corrections it provides, the use of 3D simulations was also motivated by the absence of adjustable parameters, which is not the case for 1D models.

This slightly smaller linear radius obtained from 3D RHD simulations, compared with the one derived from 1D ATLAS model, supports the suggestion of a smaller mass $(M=$ $0.907 M_{\odot}$, Kervella et al. 2003) rather than the larger one ( $\left.M=0.934 \pm 0.007 M_{\odot}\right)$ proposed by Pourbaix et al. (2002). However, stellar evolution models are sensitive to many parameters, and a smaller radius does not always lead to a smaller mass. More investigations are thus needed before we can reach a definite conclusion about the mass of $\alpha$ Cen B. In this context, our improved radius provides an additional constraint on asteroseismic diagnostics.

\section{Conclusion}

In this paper we improve determination of the radius of $\alpha$ Cen $\mathrm{B}$ in two respects. Firstly, we report the first interferometric measurements in the second lobe of visibility. Secondly, in order to derive a reliable value of the angular diameter of the star, we performed realistic 3D RHD simulations of the surface of $\alpha$ Cen B. By comparison with observations, we found a radius of $0.863 \pm 0.003 R_{\odot}$. The correction provided by the 3D approach is small but significant (especially in the $K$ band probed with VINCI), since it provides a radius that is smaller by roughly $1 \sigma_{\text {stat }}$ compared with what can be obtained by 1D models. Moreover, the use of 3D RHD is preferable since it does not introduce adjustable parameters to describe convection. We also emphasize that for hotter A-F stars the correction due to 3D analysis will be larger than for $\alpha \mathrm{Cen} \mathrm{B}$. We have shown that even for a K-dwarf like $\alpha$ Cen B, though it is small, the correction obtained by the use of RHD simulations should not be neglected for very high precision interferometric measurements. In the next few years, the combination of high visibility precision and long baselines will require the use of realistic theoretical models of the stellar limb-darkening to extract the true photospheric angular diameter of the observed stars from the observed visibilities. Conversely, observations beyond the first minimum of the visibility function will directly sample the light distribution on the surface of the stars, therefore providing constraints for the atmosphere structure models. Future observations with the VLTI will allow to sample tightly the second lobe of the visibility function of several solar analogs (including $\alpha$ Cen $\mathrm{A}$ and $\mathrm{B}$ ), and therefore to derive their intensity profiles with high accuracy. Comparisons between our theoretical predictions of limb-darkening and the future observations made by AMBER will be an excellent test of our modelling of the surface of $\alpha$ Cen B. Indeed, AMBER will provide new interferometric data that will contain much more information

error cannot be completely excluded, in particular, due to the extreme brightness and binarity of $\alpha \mathrm{Cen}$. 

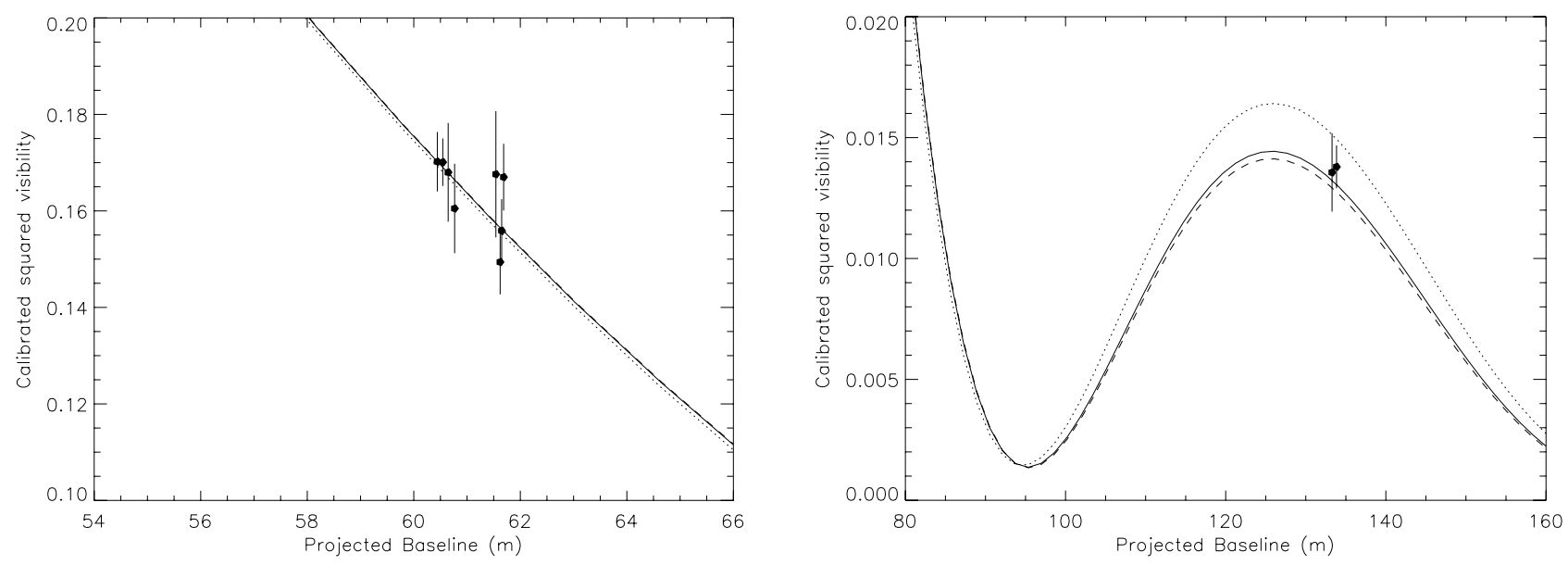

Fig. 5. Close-up views of the squared visibilities of $\alpha$ Cen B in the lower part of the first lobe (left panel) and the second lobe (right panel). The continuous line represents the broadband, limb darkened disk visibility model derived from the 3D RHD with $\theta_{3 \mathrm{D}}=6.000$ mas. The dashed lines correspond to results obtained from the 1D ATLAS model with $\theta_{1 \mathrm{D}}=6.017$ mas. The upper dotted curve is a UD model with $\theta_{\mathrm{UD}}=5.881$ mas.

compared with VINCI. There will be two major advantages with AMBER:

- It will provide a wavelength dependence of the visibility $([1.9-2.4] \mu \mathrm{m})$ therefore allowing differential studies of limb-darkening as a function of wavelength.

- AMBER can simultaneously combine the light from three telescopes and therefore measure the closure phase. This gives an advantage to determining the angular size of the star when observing in the minima of the visibility function.

These improvements will lead to better constrained angular diameters of $\alpha$ Cen $\mathrm{A}$ and B and, therefore, to high precision measurement of the ratio of the linear radii of $\mathrm{A}$ and $\mathrm{B}$, independent of the parallax.

Acknowledgements. We thank Vincent Coudé du Foresto for important remarks that led to improvements at an early stage of this paper. We thank the anonymous referee for constructive remarks. These interferometric measurements were obtained using the VLTI (ESO Paranal, Chile), and were retrieved from the ESO/ST-ECF Archive (Garching, Germany). LB thanks the CNES for financial support and $\AA$ A. Nordlund for providing his RHD code. We also thank Claude Van't Veer for providing the ATLAS model.

\section{References}

Allende Prieto, C., Asplund, M., Garcia Lòpez, R. J., \& Lambert, D. L. 2002, ApJ, 567, 544

Allende Prieto, C., Asplund, M., \& Fabiani Bendicho, P. 2004, A\&A, 423, 1109

Asplund, M., Nordlund, Å., Trampedach, R., \& Stein, R. F. 1999, A\&A, 346L, 17

Asplund, M., Ludwig, H.-G., Nordlund, Å., \& Stein, R. F. 2000a, A\&A, 359, 669

Asplund, M., Nordlund, Å., Trampedach, R., Allende Prieto, C., \& Stein, R. F. 2000b, A\&A, 359, 729

Asplund, M., Nordlund, Å., Trampedach, R., \& Stein, R. F. 2000c, A\&A, 359, 743

Asplund, M., Grevesse, N., Sauval, A. J., Allende Prieto, C., \& Kiselman, D. 2004, A\&A, 417, 751
Asplund, M., Grevesse, N., Sauval, A. J., Allende Prieto, C., \& Blomme, R. 2005, A\&A, 431, 693

Bordé, P., Coudé du Foresto, V., Chagnon, G., \& Perrin, G. 2002, A\&A, 393, 183

Bouchy, F., \& Carrier, F. 2001, A\&A, 374, L5

Bouchy, F., \& Carrier, F. 2002, A\&A, 390, 205

Carrier, F., \& Bourban, G. 2003, A\&A, 406, 23

Claret, A. 2000, A\&A, 363, 1081

Cohen, M., Walker, R. G., Carter, B., et al. 1999, AJ, 117, 1864

Eggenberger, P., Charbonnel, C., Talon, S., et al. 2004, A\&A, 417, 235

Gustafsson, B., Bell, R. A., Eriksson, K., \& Nordlund, Å. 1975, A\&A, 42, 407

Hauschildt, P. H., Allard, F., \& Baron, E. 1999, ApJ, 512, 377

Hestroffer, D., \& Magnant, C. 1998, A\&A, 333, 338

Kervella, P., Coudé du Foresto, V., Glindemann, A., \& Hofmann, R. 2000, SPIE, 4006, 31

Kervella, P., Gitton, Ph., Ségransan, D., et al. 2003a, SPIE, 4838, 858

Kervella, P., Thévenin, F., Ségransan, D., et al. 2003b, A\&A, 404, 1087 (Paper I)

Kervella, P., Ségransan, D., \& Coudé du Foresto, V. 2004, A\&A, 425, 1161

Kurucz, R. L. 1992, The Stellar Populations of Galaxies, IAU Symp., 149,225

Ludwig, H., Allard, F., \& Hauschildt, P. H. 2002, A\&A, 395, 99

Morel, P., Provost, J., Lebreton, Y., Thévenin, F., \& Berthomieu, G. 2000, A\&A, 363, 675

Neckel, H., \& Labs, D. 1994, SoPh, 153, 91

Nordlund, Å. 1982, A\&A, 107, 1

Nordlund, Å., \& Dravins, D. 1990, A\&A, 228, 155

Petrov, R. G., Malbet, F., Richichi, A., et al. 2000, SPIE, 4006, 68

Pierce, A. K., \& Slaughter, C. D. 1977, SoPh, 51, 25

Pourbaix, D., Nidever, D., McCarthy, C., et al. 2002, A\&A, 386, 280

Robbe-Dubois, S., Petrov, R. G., Lagarde, S., et al. 2004, SPIE, 5491, 1089

Rosenthal, C. S., Christensen-Dalsgaard, J., Nordlund, Å., Stein, R. F., \& Trampedach, R. 1999, A\&A, 351, 689

Stein, R. F., \& Nordlund, ̊. 1989, ApJ, 342, L95

Stein, R. F., \& Nordlund, A.. 1998, ApJ, 499, 914

Söderhjelm, S. 1999, A\&A, 341, 121

Thévenin, F., Provost, J., Morel, P., et al. 2002, A\&A, 392, L9

Thoul, A., Scuflaire, R., Noels, A., et al. 2003, A\&A, 402, 293 